\title{
68Ga-PSMA PET/MR-positive, histopathology-proven prostate cancer in a patient with negative multiparametric prostate MRI
}

\author{
Muehlematter, Urs J ; Rupp, Niels J ; Mueller, Julia ; Eberli, Daniel ; Burger, Irene A
}

\begin{abstract}
Multiparametric MRI incorporating T2-weighted, diffusion-weighted, and dynamic contrast material-enhanced sequences is currently used for detection and localization of clinically important prostate cancer $(\mathrm{PCa})$. The Ga-labeled PET tracer targeting the prostate-specific membrane antigen (PSMA, Ga-PSMA-11) is a promising diagnostic approach for staging and restating PCa. Recent studies suggest that Ga-PSMA could also be used for primary PCa detection and localization. We report a case of a Ga-PSMA PET/MR-positive lesion of the peripheral zone in a 73-year-old man with a negative preceding multiparametric MRI. Radical prostatectomy and subsequent histopathologic examination confirmed a Gleason $4+4$ PCa.
\end{abstract}

DOI: https://doi.org/10.1097/RLU.0000000000002143

Posted at the Zurich Open Repository and Archive, University of Zurich ZORA URL: https://doi.org/10.5167/uzh-152849

Journal Article

Published Version

Originally published at:

Muehlematter, Urs J; Rupp, Niels J; Mueller, Julia; Eberli, Daniel; Burger, Irene A (2018). 68Ga-PSMA PET/MR-positive, histopathology-proven prostate cancer in a patient with negative multiparametric prostate MRI. Clinical Nuclear Medicine, 43(8):e282-e284.

DOI: https://doi.org/10.1097/RLU.0000000000002143 


\title{
${ }^{68}$ Ga-PSMA PET/MR-Positive, Histopathology-Proven Prostate Cancer in a Patient With Negative Multiparametric Prostate MRI
}

\author{
Urs J. Muehlematter, MD, * Niels J. Rupp, MD, † Julian Mueller, MD, + \\ Daniel Eberli, MD, PhD, \& and Irene A. Burger, MD
}

\begin{abstract}
Multiparametric MRI incorporating T2-weighted, diffusionweighted, and dynamic contrast material-enhanced sequences is currently used for detection and localization of clinically important prostate cancer (PCa). The ${ }^{68}$ Ga-labeled PET tracer targeting the prostate-specific membrane antigen (PSMA, ${ }^{68} \mathrm{Ga}$-PSMA-11) is a promising diagnostic approach for staging and restating PCa. Recent studies suggest that ${ }^{68} \mathrm{Ga}$-PSMA could also be used for primary PCa detection and localization. We report a case of a ${ }^{68}$ Ga-PSMA PET/MR-positive lesion of the peripheral zone in a 73-yearold man with a negative preceding multiparametric MRI. Radical prostatectomy and subsequent histopathologic examination confirmed a Gleason $4+4$ PCa.
\end{abstract}

Key Words: ${ }^{68} \mathrm{Ga}$-PSMA, PET/MRI, prostate cancer, multiparametric MRI (Clin Nucl Med 2018;43: e282-e284)

Received for publication February 25, 2018; revision accepted April 7, 2018 From the Departments of *Diagnostic and Interventional Radiology, $\uparrow$ Pathology and Molecular Pathology, \$Nuclear Medicine, and §Urology, University Hospital Zurich, University of Zurich, Zurich.

Conflicts of interest and sources of funding: none declared.

Correspondence to: Urs J. Muehlematter, MD, Department of Diagnostic and Interventional Radiology, University Hospital Zurich, University of Zurich, Rämistrasse 100, 8091 Zürich, Switzerland. E-mail: urs.muehlematter@usz.ch. Copyright $(C 2018$ Wolters Kluwer Health, Inc. All rights reserved.

ISSN: 0363-9762/18/4308-e282

DOI: $10.1097 / R L U .0000000000002143$

\section{REFERENCES}

1. Weinreb JC, Barentsz JO, Choyke PL, et al. PI-RADS prostate imagingreporting and data system: 2015, version 2. Eur Urol. 2016;69:16-40.

2. Eiber M, Nekolla SG, Maurer T, et al. (68)Ga-PSMA PET/MR with multimodality image analysis for primary prostate cancer. Abdom Imaging. 2015;40:1769-1771.

3. Perner S, Hofer MD, Kim R, et al. Prostate-specific membrane antigen expression as a predictor of prostate cancer progression. Hum Pathol. 2007; 38:696-701.

4. Hoeks CM, Barentsz JO, Hambrock T, et al. Prostate cancer: multiparametric MR imaging for detection, localization, and staging. Radiology. 2011;261: 46-66.

5. Boesen L, Chabanova E, Løgager V, et al. Apparent diffusion coefficient ratio correlates significantly with prostate cancer Gleason score at final pathology. J Magn Reson Imaging. 2015;42:446-453.

6. Oto A, Kayhan A, Jiang Y, et al. Prostate cancer: differentiation of central gland cancer from benign prostatic hyperplasia by using diffusion-weighted and dynamic contrast-enhanced MR imaging. Radiology. 2010;257:715-723.

7. Simpkin CJ, Morgan VA, Giles SL, et al. Relationship between $T_{2}$ relaxation and apparent diffusion coefficient in malignant and non-malignant prostate regions and the effect of peripheral zone fractional volume. $\mathrm{Br} J$ Radiol. 2013;86:20120469.

8. Zelhof B, Pickles M, Liney G, et al. Correlation of diffusion-weighted magnetic resonance data with cellularity in prostate cancer. BJU Int. 2009;103: 883-888.

9. Filson CP, Natarajan S, Margolis DJ, et al. Prostate cancer detection with magnetic resonance-ultrasound fusion biopsy: The role of systematic and targeted biopsies. Cancer. 2016;122:884-892.

10. Borofsky S, George AK, Gaur S, et al. What are we missing? False-negative cancers at multiparametric MR imaging of the prostate. Radiology. 2017; 286:186-195.

11. Eiber M, Weirich G, Holzapfel K, et al. Simultaneous ${ }^{68}$ Ga-PSMA HBED-CC PET/MRI improves the localization of primary prostate cancer. Eur Urol. 2016;70:829-836.

12. Simopoulos DN, Natarajan S, Jones TA, et al. Targeted prostate biopsy using ${ }^{68}$ Gallium PSMA-PET/CT for image guidance. Urol Case Rep. 2017;14: 11-14. 

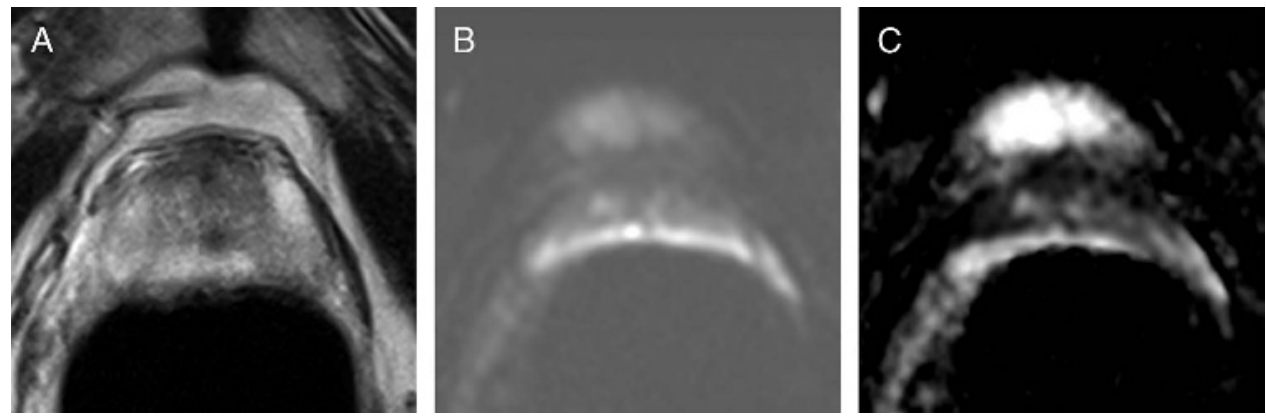

FIGURE 1. Multiparametric MRI (mpMRI). A 73-year-old man with newly diagnosed surge of prostate-specific antigen from 2.15 to $2.85 \mathrm{ng} / \mathrm{mL}$. Transverse T2-weighted images (A) show slight hypointense signal in the peripheral zone basal posterior on both sides. Prostate Imaging Reporting and Data System (PI-RADS) version $2^{1}$ using information from diffusion-weighted imaging (B) apparent diffusion coefficient (ADC) map (shown in C) and dynamic contrast-enhanced imaging (not shown) resulted in a PI-RADS scores of 2 for the right and left basal sextant. However, rectal gas limited image interpretation, especially because of artifacts in the diffusion-weighted images.
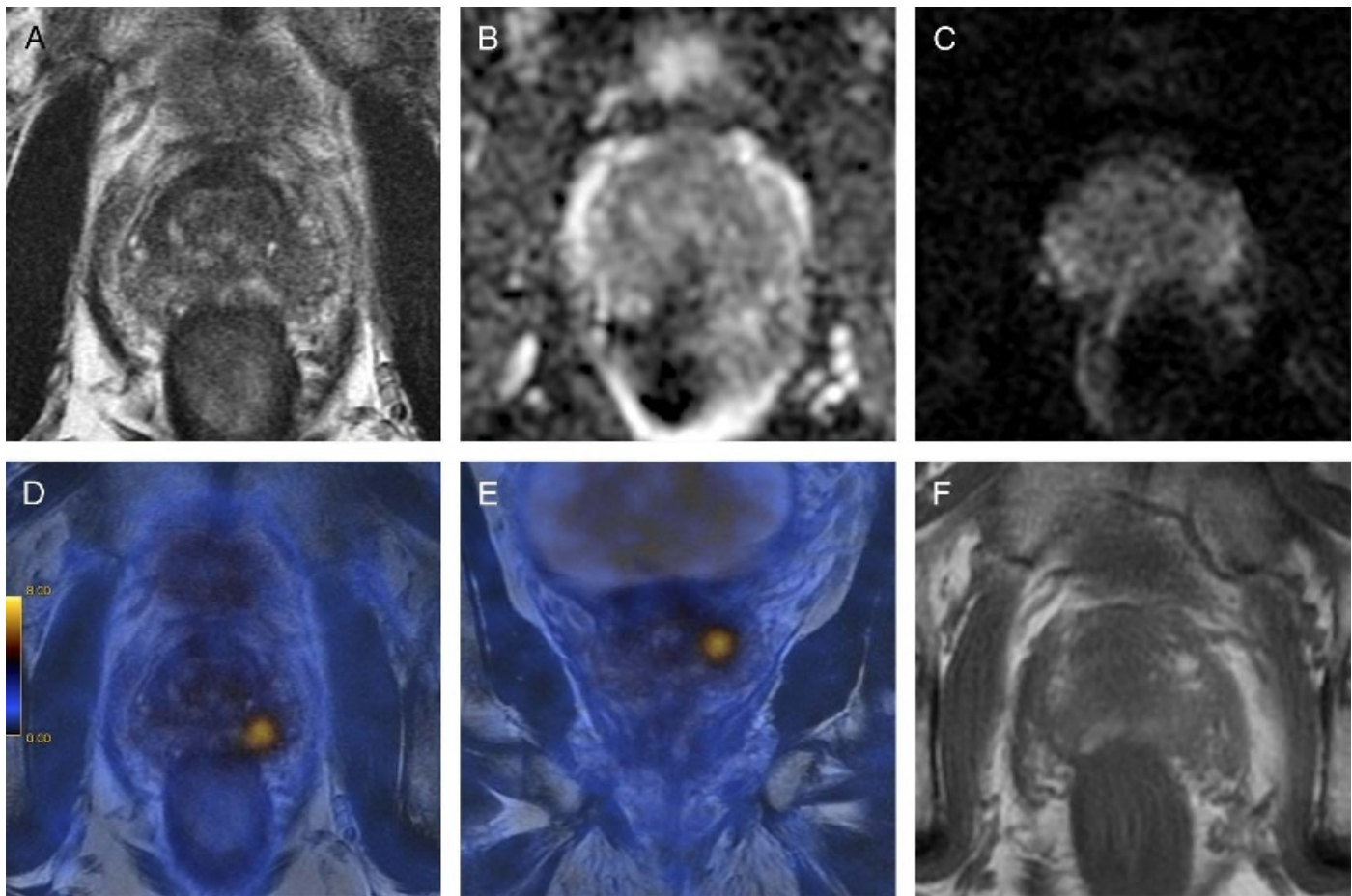

FIGURE 2. ${ }^{68} \mathrm{Ga}$ prostate-specific membrane antigen (PSMA) PET/MRI scan 1 month after a template biopsy and 8 months after the initial mpMRI of the prostate. Template biopsy revealed an adenocarcinoma of the prostate with Gleason score (GS) $4+4=8$ in 2 of 33 cores. Prostate-specific antigen level was $3.82 \mathrm{ng} / \mathrm{mL}$ at scan time of the PET/MRI. Transverse T2-weighted images (A) show diffuse hypointense signal in the peripheral zone basal posterior on both sides, diffusion-weighted imaging (B) and ADC map shown in (C) do not demonstrate restricted diffusion. Axial (D) and coronal fused (E) ${ }^{68}$ Ga-PSMA PET/MRI scans show a single PSMA-positive prostatic lesion of $6 \times 6 \times 6 \mathrm{~mm}$ in the left basal sextant with an SUV max $_{\text {Of }} 6.9$. There was no evidence of local or distant metastasis. T1-weighted image (F) shows several postbioptic changes that are reported not to influence the PET. ${ }^{2}$ 
A

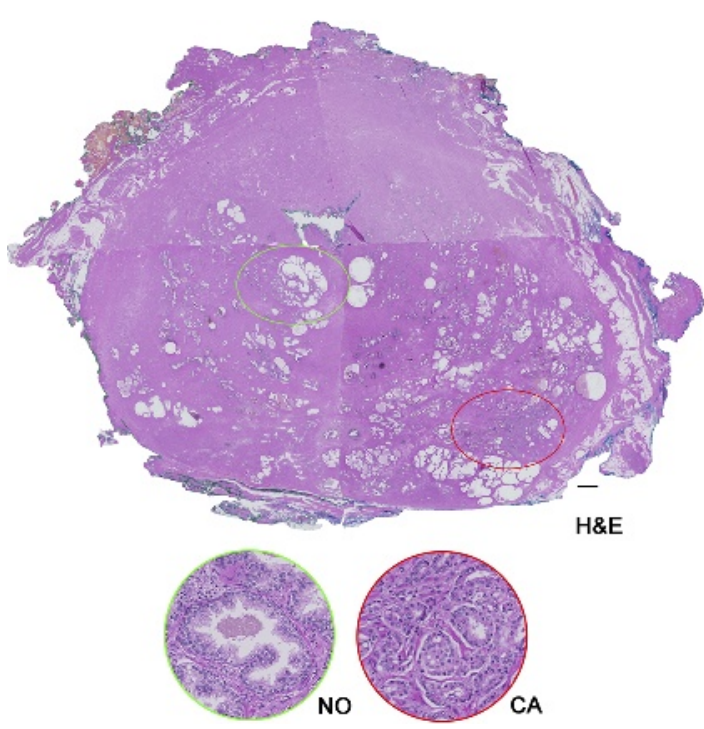

B

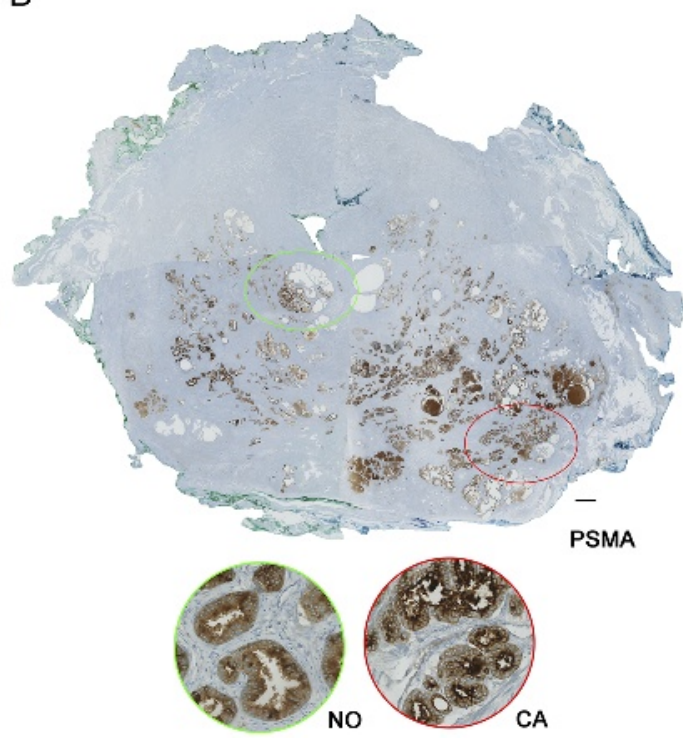

FIGURE 3. Reconstructed histopathological slices of the corresponding prostate after radical prostatectomy and pelvic lymph node (LN) dissection. H\&E-stained (A) and PSMA-stained slice (B) show a GS $4+4$ prostate cancer (PCa) of the left basal sextant that match the PSMA-positive lesion. Note the mainly membranous staining in PCa, whereas the benign tissue (NO) shows a predominant cytoplasmic staining ${ }^{3}$ (insets). No positive LN was found in the LN dissection specimen. Despite multiple publications showing a good correlation between mpMRI with ADC and higher $G S P C a,{ }^{4-8}$ cases with clinically relevant PCa and false-negative mpMRI have been reported. ${ }^{9,10}$ More recently, cases with clinically relevant PCa, false-negative mpMRI, and true-positive PSMA PET have been reported. ${ }^{11,12}$ PSMA PET/MR has been described as a potential tool for staging of high-risk PCa with improved sensitivity for LN and distant metastasis. ${ }^{2}$ In our case, PSMA PET/MR clearly delineated the highly

aggressive tumor, not detected on mpMRI and therefore improved the sensitivity for the local tumor. Furthermore, $\mathrm{LN}$ and distant metastasis could be ruled out. PSMA PET/MR might be used as a "trouble shooter" in selected cases with discrepancies between mpMRI and biopsy results. 